\title{
Etapas na Otimização Experimental de Produtos e Processos: Discussão e Estudo de Caso
}

\author{
Carla S. ten Caten
}

Mestre em Engenharia de Produção - PPGEP-UFRGS

José Luis Duarte Ribeiro

Doutor em Engenharia - PPGEP-UFRGS

Programa de Pós-Graduação em Engenharia de Produção

Escola de Engenharia - UFRGS

Praça Argentina n0 9, sala 402 - POA - RS

Palavras-chave: Qualidade, Otimização, Projeto de Experimentos, Regressão Múltipla, Função de Perda.

Key words: Quality, Optimization, Design of Experiments, Multiple Regression, Loss Function.

\section{RESUMO}

Este artigo descreve um método de otimização experimental de produtos ou processos medidos por múltiplas variáveis de resposta. Todas as etapas do método, que contempla desde a identificação do problema até a otimização de custos e qualidade, são descritas em detalhe.

Para ilustrar a aplicação do método, relata-se um estudo de otimização de um processo de fundição. Este estudo de caso foi inicialmente reportado por Chang e Shivpuri (1995). Este artigo apresenta uma comparação dos resultados obtidos por essses autores e aqueles obtidos utilizando-se o método proposto. As vantagens do método proposto são evidenciadas.

\footnotetext{
ABSTRACT

This paper presents a method for the optimization of processes or products measured by multiple responses. All the steps of the method, which covers from problem identification to quality and costs optimization, are described.

To illustrate the use of the method, an optimization study conducted on a casting process is discussed. This case study was first reported by Chang e Shivpuri (1995). This paper presents a comparison of the results obtained by these authors and those obtained through the use of the proposed method. The advantages of the proposed method are discussed and pointed out.
} 


\section{PRODUÇÃO}

\section{Introdução}

Este trabalho descreve um método de otimização experimental de produtos ou processos. O método visa, prioritariamente, a otimização da qualidade em sistemas avaliados por múltiplas variáveis de resposta. Algumas das vantagens do método são sua simplicidade e flexibilidade. Além de considerar múltiplas variáveis de resposta, $o$ método acessa múltiplos objetivos, que podem ser otimizados simultaneamente.

O método proposto neste artigo desdobra-se em cinco etapas principais:

(1) Identificação do problema,

(2) Planejamento e execução de experimentos,

(3) Modelagem individual das variáveis de resposta,

(4) Definição de uma função objetivo,

(5) Otimização.

A etapa de identificação do problema é importante para se definir corretamente o objetivo do estudo e fazer um planejamento adequado do experimento a ser usado para a coleta de dados. A partir dos resultados dos experimentos, faz-se uma modelagem individual das variáveis de resposta, ajustando-se um modelo para a média e outro para o desviopadrão de cada uma das variáveis de resposta (em função dos fatores controláveis). Esse passo permite ampliar o conhecimento sobre o sistema em estudo. Conhecendo-se os modelos individuais para as múltiplas variáveis de resposta, eles são incorporados em uma função objetivo, que pondera a importância relativa de cada uma dessas variáveis. Dessa forma, pode-se trabalhar um problema complexo, com múltiplas variáveis de resposta, na forma de um problema simples, com uma única resposta a otimizar (no caso o valor da função objetivo). Além de incorporar as várias variáveis de resposta, a função objetivo proposta incorpora diferentes objetivos. Assim, o procedimento de otimização torna-se mais abrangente.

Este trabalho está organizado ell duas seções principais: a seção 2, que apresenta a descrição do método, e a seção 3 , que apresenta um estudo de caso, exemplificando a aplicação do método proposto.

\section{Descrição do Método}

Esta seção apresenta em detalhe as cinco etapas do método proposto. As etapas I e 2 foram escritas a partir da compilação das idéias de Box, Hunter \& Hunter (1978), Colemann \& Montgomery (1993), Hahn (1977), Montgomery (1991) e Nanni e Ribeiro (1991). A etapa 3 , trata da modelagem de variáveis de resposta, prática comum dentro da engenharia da qualidade. A etapa 4 baseia-se na proposta de Ribeiro e Elsayed (1995), enquanto que a etapa 5 utiliza-se de procedimentos comuns de otimização de funções. 


\section{PRODUÇÃO}

\section{Etapa 1 - Identificação do Problema}

Nessa etapa, é interessante trabalhar com uma equipe multidisciplinar, que reúna pessoas de vários setores. Dessa forma, é possível obter o máximo de informações sobre o problema que será estudado, definindo o objetivo do estudo de maneira que atenda a todos os interessados.

Os passos necessários para a correta identificação do problema são os seguintes:

\section{Identificar claramente os objetivos do estudo}

Os objetivos devem ser especificos, mensuráveis, não tendenciosos e devem ter consequiência prática. Para isso, a equipe técnica deve contar com a participação de todas as pessoas interessadas no experimento, para estabelecer e detalhar o objetivo de tal maneira que fique claro quando este foi alcançado. A realização de um programa experimental implica gastos, por isso o resultado obtido deve ter consequiência prática, que represente uma melhoria do produto ou processo.

Identificar as características de qualidade de interesse dos consumidores e engenheiros

O estudo deve ser dirigido tendo como foco o cliente. Assim, é essencial conhecer quais as caracteristicas de qualidade de interesse para o cliente. $O$ uso de pesquisa de mercado e consulta ao corpo técnico da empresa devem ser empregados para completar essa fase, permitindo a correta associação das características que interessam ao cliente com as variáveis do processo em estudo. A consulta ao corpo técnico, referente às características de qualidade pertinentes ao produto, também é importante, pois muitas vezes, o corpo técnico pode incorporar características que não são identificadas pelo consumidor.

Eleger as variáveis de resposta, associadas a cada característica de qualidade

Nesse passo escolhem-se as variáveis de resposta a serem utilizadas no estudo experimental. As variáveis de resposta devem quantificar objetivamente as características de qualidade, que muitas vezes podem ser aspectos vagos e subjetivos. Idealmente, uma variável de resposta deveria ser contínua, capturar a quantidade ou qualidade de interesse para o estudo experimental, estar em unidades apropriadas e associada com um valor alvo desejável, ser obtida por ensaios nãodestrutivos, não estar próxima dos limites naturais, ter variância constante sobre toda a amplitude de experimentação, ser fácil de medir e ter uma relação direta com o objetivo do experimento. Nessa fase, as especificações, o valor alvo e a importância relativa de cada uma das variáveis de resposta devem ser corretamente identificados.

A avaliação da importância relativa das variáveis de resposta é feita por consenso, considerando a opinião de todos os envolvidos com o produto ou processo. 


\section{PRODUÇÃO}

Etapa 2 - Planejamento e Execução de Experimentos

Para obtermos dados sobre o problema a ser estudado, é recomendável estruturar a coleta de dados usando a metodologia de projeto de experimentos. O uso dessa metodologia possibilita o planejamento de experimentos eficientes e econômicos e com resultados confiáveis.

Dado que o objetivo do estudo já tenha sido corretamente especificado, os passos necessários para um bom planejamento do experimento são os seguintes:

Identificar todos os parâmetros do processo que afetam as variáveis de resposta

Esse passo exige conhecimento técnico do processo ou produto. Todos aqueles parâmetros do processo ou do produto que possam afetar as variáveis de resposta devem ser listados. Então é preciso decidir como tratá-los no experimento. Existem quatro maneiras de trabalhar com os parâmetros do processo: fatores principais, fatores secundários, fatores mantidos constantes e fatores não controláveis, sendo que a essolha de como trabalhar com eles envolve um compromisso entre custo, tempo e informação.

Identificar os fatores principais e os fatores secundários, ou seja, os fatores controláveis.

Oś fatores controláveis são o subconjunto dos parâmetros do processo/ produto que será estudado (ensaiado a vários níveis) durante o experimento. Os fatores principais são aquelas fatores que podem ser controlados pelos engenheiros e que podem ter um efeito sobre as variáveis de resposta. Os fatores secundários são aqueles que, embora não sejam de interesse principal, também podem exercer efeito sobre a resposta e não deveria ser possível mantê-los constantes. É muito importante que os fatores secundários não variem junto com os fatores principais, caso contrário seus efeitos podem resultar confundidos.

\section{Identificar os fatores a serem mantidos constantes \\ Aqueles parâmetros do processo/ produto que podem ser controlados, mas que possivelmente não tenham efeito significativo sobre a resposta (ou cujo efeito já é conhecido), devem ser mantidos constantes durante o experimento. Assim, o tamanho, o custo e a complexidade do experimento ficam reduzidos.}

Identificar os fatores de ruido e os procedimentos para diminuir seu efeito

Os fatores de ruido são os fatores não controláveis que podem ter algum efeito sobre as variáveis de resposta. Eles são os responsáveis pelo erro experimental e diferem dos fatores mantidos constantes, pois não podem ser fixados a um nivel constante para todas as rodadas. Se um nivel constante pode ser selecionado para qualquer unidade experimental, o uso de blocagem e aleatorização pode ser apropriado. Se os niveis não podem ser selecionados, então os fatores de ruído tornam-se uma covariável. Se os fatores 
de ruído não são mensuráveis e são muito influentes, eles podem ser chamados de "fatores de risco experimental". Tais fatores podem inflacionar o erro experimental de tal maneira que fique difícil avaliar a significância dos fatores controláveis, podendo levar a uma distorção do resultado. Por isso, os experimentadores devem se preocupar em determinar uma estratégia para reduzir o impacto dos fatores de ruído. Essas estratégias podem ser aleatorização, blocagem, análise de covariância, análise estratificada e outras mais.

Identificar a eventual existência de interaçōes entre os fatores controláveis

Se houver interações entre os fatores controláveis, scus efeitos deverão ser estudados no experimento. Esses dados são úteis na escolha do modelo estatístico e da resolução do experimento. A resolução define quais os termos (interações) que podem ficar confundidos em experimentos fatoriais fracionados. Quanto maior a resolução, maior a precisão da estimativa dos efeitos, pois um menor número de efeitos estará confundido. Se os fatores secundários não interagem com os fatores principais, eles podem ser introduzidos no experimento na forma de blocos experimentais. A principal razão para rodar um experimento em bloco é assegurar que o efeito dos fatores secundários não contaminem a avaliação dos fatores principais. A blocagem permite que o efeito dos fatores blocados seja removido do erro cxperimental, fornecendo uma avaliação mais precisa da significância dos fatores principais. Por último, em muitas situações, o efeito dos fatores blocados sobre a variável de resposta pode ser avaliado.

\section{Definir o intervalo de estudo dos fatores controláveis}

A escolha da amplitude do intervalo dos fatores controláveis deve ser feita com cuidado, a fim de permitir a investigação de toda a região de interesse. Essa escolha deve ter como base o conliecimento da amplitude operacional que pode ser atingida e dos valores de uso corrente. A amplitude escolhida deve cobrir uma boa proporção da amplitude operacional e produzir um efeito observável. Contudo, não pode ser tão grande a ponto de impedir o ajuste de qualquer modelo empírico para a região escolhida. A escolha dos niveis altos/baixos dos fatores controláveis deveria produzir um efeito sobre alguma variável de resposta que fosse superior a um desvio-padrão de sua variação no uso comum. Esse efeito é suficientemente grande para ter consequiência prática e detectar se os erros de medição são despreziveis.

\section{Definir o número de níveis dos} fatores controláveis

O número de níveis deve ser escollhido considerando o provável efeito do fator controlável sobre as variáveis de resposta, o qual pode ser linear (ao menos dois níveis), quadrático (ao menos três niveis) ou de ordem superior (mais do que três niveis). 


\section{PRODUÇÃO}

Considerar as restrições experimentais

As restrições podem ser o número máximo de ensaios, dificuldades na mudança nos niveis dos fatores controláveis, tempo disponível, e outras que devem ser incorporadas no modelo estatístico do experimento.

Avaliar as vantagens e desvantagens de rodadas prévias

Rodadas prévias, que antecedem o experimento em si, podem ser importantes, especialmente quando se trata da primeira fase de um programa experimental. Em estudos de otimização, a realização de experimentos em estágios é atrativa, pois permite mover a região de investigação de estágio para estágio. Por outro lado, um experimento realizado de uma única vez pode ser desejável no caso de altos custos de preparação para cada estágio, ou quando há um longo tempo de espera entre a fabricação das unidades e a medida de sua performance. Se o experimento for realizado em estágios, precauções devem ser tomadas para assegurar que as diferenças possiveis entre os estágios não invalidem o experimento. Um motivo das rodadas prévias é apreender e refinar os procedimentos experimentais, sem arriscar a perda de tempo e amostras experimentais caras. Um segundo motivo é estimar o erro experimental antes de gastar maiores recursos, pois um erro experimental grande pode levar ao cancelamento ou reprojeto do experimento. Pode ser necessário aumentar a amplitude dos niveis, aumentar o número de replicações ou refinar os procedimentos de ensaio. Um terceiro motivo é que as rodadas prévias são excelentes oportunidades para assegurar que o sistema de aquisição de dados esteja funcionando e que as rodadas posteriores serão conduzidas conforme planejado.

\section{Estimar a variabilidade das variáveis de resposta previamente \\ Estimativas preliminares da variabilidade} da resposta sob as mesmas condições experimentais deveriam ser obtidas antes de realizar-se um experimento em larga escala, pois valores altos da variabilidade indicam que a significância de fatores que afetam a variável de resposta podem terminar não sendo identificada. Quanto maior a precisão desejada para a estimativa, maior será o número de rodadas experimentais exigidas. Procedimentos para documentação dos dados devem ser estabelecidos, pois deve-se anotar as condições reais na qual o experimento foi rodado e se houve algum fator não planejado que pode ter influenciado a resposta. Os valores dessas "covariáveis" podem ser fatorados na análise estatística, conseqüentemente reduzindo a variabilidade não quantificada ou o erro experimental.

\section{Definir o modelo de experimento a ser realizado}

A definição do modelo deve ser feita levando em conta todos os aspectos mencionados anteriormente, além de outras restrições (financeiras ou técnicas) que, em geral, existem.

O planejamento experimental final deve incorporar o melhor compromisso entre custo e informação pois, além de fornecer resultados válidos estatisticamente, o 
experimento deve ser prático de executar. Para o sucesso do experimento é necessário um bom planejamento, realizado em conjunto por estatísticos e engenheiros. Esse planejamento deve passar pelas fases descritas anteriormente.

Uma primeira estimativa dos efeitos pode ser pesquisada na literatura, ou pode ser obtida com especialistas. A tentativa de predizer o resultado frequientemente provoca a revisão da escolha dos niveis dos fatores controláveis e proporciona a comparação do conhecimento ganho com o experimento com a intuição dos experimentadores.

Segundo Hahn (1977), projetar um experimento é um processo iterativo, exigindo retrabalho sempre que surgem informações novas ou quando os dados preliminares estão disponíveis. Com um entendimento global do problema, os estatísticos adquirem uma posição privilegiada para responder rapidamente caso mudanças sejam necessárias e para fornecer análises importantes sobre os resultados experimentais subsequientes.

\section{Etapa 3 - Modelagem Individual das Variáveis de Resposta}

Nesta etapa, procede-se a uma modelagem individual de cada variável de resposta em função dos fatores controláveis. A modelagem pode ser feita, por exemplo. utilizando-se rotinas de regressão linear ou não-linear. Os modelos possibilitam identificar quais fatores interferem em quais variáveis de resposta. Fazendo isso, a equipe tem a oportunidade de aprender importantes aspectos sobre o problema em estudo.

Além da média, também a variabilidade das variáveis de resposta pode ser modelada em função dos fatores controláveis. Com isso, pode-se ajustar os fatores controláveis em niveis que minimizem a variabilidade.

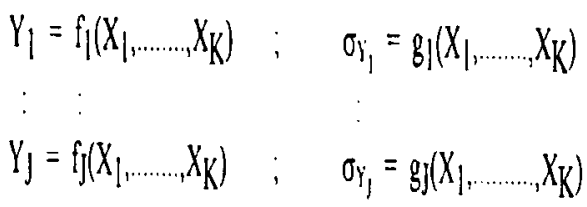

As estimativas dos valores médios são úteis para identificar tratamentos próximos do alvo, enquanto que as estimativas da variabilidade ajudam na identificação de tratamentos robustos ao ruído, ou seja, menos sensiveis aos efeitos dos fatores não-controláveis.

Essas estimativas de média e desvio padrão serão inseridas na função objetivo que será utilizada na otimização. Quando trabalha-se com modelos para a media e a variância, o erro da estimativa é menor, pois trabalha-se com valores médios e não com valores individuais. Logo, o erro na estimativa da perda associada a um certo tratamento é menor.

Além de reduzir os erros associados ao ajuste de um modelo para a função de perda, a estratégia de modelagem individual das variáveis de resposla possibilita um maior conhecimento do processo. Vale ressaltar que a etapa de modelagem qualifica o estudo de otimização, uma vez 
que os modelos capturam a tendência presente nos dados e permitem efetuar previsões. Dessa forma, as estimativas das variáveis de resposta consideram a informação contida em todos os resultados do experimento.

Conforme o caso, os modelos podem incluir efeitos lineares dos fatores controláveis, efeitos quadráticos, interações de dois fatores ou efeitos não lineares mais complexos. Com isso, as estimativas das variáveis de resposta ficam mais precisas.

Os modelos obtidos para as variáveis de resposta devem conter apenas os termos que apresentam efeito significativo. A significância do efeito dos diversos termos do modelo é verificada, por exemplo, através da estatistica " $t$ " ou da probabilidade " $p$ " fornecida nas rotinas de regressão múltipla ou na ANOVA.

A identificação de fatores controláveis que não tenham efeito significativo sobre as variáveis de resposta é igualmente imporlante, pois para esses fatores controláveis escollheml-se níveis que são mais econômicos. Dessa forma, maximiza-se a qualidade, através dos fatores controláveis com efeito significativo, e minimiza-se o custo (de operação, de matérias primas), através dos fatores controláveis que não tenham efeito significativo.

Outra grande vantagem da modelagem, é o fato de se poder estimar o valor das variáveis de resposta para niveis intermediários dos fatores controláveis. Assim, a estimativa das variáveis de resposta não fica restrita aos niveis dos fatores controláveis que foram ensaiados no experimento. Pode-se fazer estimativas das variáveis de resposta para qualquer nivel dentro do intervalo ensaiado no experimento (interpolação) e, com alguma reserva, também podem ser feitas extrapolações.

\section{Etapa 4-Definir uma Função Objetivo}

Com a modelagem individual das variáveis de resposta, pode-se quantificar o efeito dos fatores controláveis (parâmetros do processo/produto) sobre cada variável de resposta. Desta forma, pode-se identificar qual o ajuste ótimo dos fatores controláveis para cada variável de resposta separadamente.

No entanto, muitas vezes, um certo ajuste dos fatores controláveis pode melhorar uma determinada variável de resposta e piorar outras. Raramente o mesmo ajuste dos fatores controláveis conduz ao valor ideal de todas as variáveis de resposta simultaneamente. Uma vez que haja conflito, é necessário fazer-se uma otimização global das variáveis de resposta. A otimização global consiste em identificar o ajuste dos fatores controláveis que otimiza simultaneamente todas as variáveis de resposta de interesse do cliente. Essa otimização global implica compromissos entre as variáveis de resposta, ou seja, uma solução conciliatória que pondere as importâncias relativas de cada variável de resposta sobre o produto final. 


\section{PRODUÇÃO}

Em estudos de melhoria da qualidade, é desejável que a otimização global considere três objetivos distintos. O primeiro consiste em fazer com que as respostas medidas sobre o produto ou processo fiquem o mais próximo possivel do seu valor ideal, ou seja, dos valores especificados no projeto, que coincidem com a demanda dos clientes.

O segundo objetivo consiste em maximizar a robustez aos fatores de ruído, ou seja, escolher niveis dos fatores controláveis que minimizem a variabilidade das variáveis de resposta devido aos fatores de ruído (temperatura, umidade, etc.). Isso significa dizer que o produto ou processo será robusto e terá um bom desempenho mesmo quando submetido a condições adversas do meio.

O terceiro objetivo consiste em tornar o produto ou processo robusto a oscilações nos próprios fatores controláveis pois, muitas vezes, na linha de produção, é dificil manter os fatores controláveis fixos em seus niveis pré-determinados. Robustez ell relação aos fatores controláveis significa obter ajustes que fornecerão valores adequados das variáveis de resposta mesmo quando ocorrem oscilações em torno do ajuste definido.

Nesta etapa é preciso definir uma função objetivo que.incorpore os diferentes objetivos mencionados anteriormente, ou seja, (i) desvios do alvo, (ii) robustez ao ruído, e (iii) robustez a oscilações nos fatores controláveis. Além disso, essa função deve levar em conta a importância relativa das diferentes variáveis de resposta.

Para realizar a otimização global, sugere-se o uso da Função de Perda Quadrática Multivariada (ver a seguir eq. 2 ), a qual busca avaliar a perda incorrida ao cliente devida aos três aspectos mencionados anteriormente. Esta perda aumenta quadraticamente à medida que a variável de resposta afasta-se do seu valor nominal. A Função de Perda Quadrática Multivariada foi inicialmente proposta por Ribeiro e Elsayed (1995).

Esta função de perda $Z$ consiste de:

$\hat{Z}(i)=\sum_{j=1}^{J} w\left[w_{j}\left[\left(\hat{Y}_{j}-T_{j}\right)^{2}+\hat{\sigma}_{Y j}^{2}+\sum_{k=1}^{K} \hat{\sigma}_{J k}^{2}\left(\frac{\hat{\partial}_{j}}{\hat{A}_{k}}\right)^{2}\right]\right.$

onde:

$\hat{Z}^{(i)}$ é a função objetivo a ser minimizada; para cada ajuste dos fatores controláveis (tratamento $i$ ), pode-se obter um valor estimado de $Z$ (i);

$w_{j}$ são pesos que levam em conta as unidades e a importância relativa de cada variável de resposta;

$T_{j}$ é o valor ideal para a variável de resposta $j$;

$\hat{Y}_{j}$ estimativa da média da variável de resposta $j$;

$\hat{\sigma}_{Y_{j}}$ estimativa do desvio padrão da variável de resposta $j$; 
$\hat{\sigma}_{X_{k}}$ estimativa do desvio padrão do fator controlável $k$.

Esta versão da função de perda referese a variáveis de resposta do tipo nominal-é-melhor. O valor da perda é mínimo quando a média do processo (valor médio) coincide com o valor ideal, e a perda aumenta simetricamente (ou não) à medida que se afasta do valor ideal. Além desse caso, existem outros tipos de variáveis de resposta. Por exemplo, caracteristicas do tipo menor-é-melhor e maior-é-melhor, usadas quando o valor ideal para a variável de resposta em estudo é respectivamente zero ou infinito.

O valor da função de perda $Z$ é diretamente proporcional aos custos gerados pela má qualidade do produto ou processo. Caso uma constante de proporcionalidade $K$ possa ser definida, as perdas devido à má qualidade poderiam ser calculadas em unidades monetárias:

$$
C_{Q}(i)=K \times Z(i)
$$

Por outro lado, também pode ser possivel modelar as perdas associadas à matéria prima e energia gastas durante o processo de manufatura, em função dos fatores controlíveis:

$$
C_{M}(i)=f\left(X_{1}, \ldots \ldots, X_{K}\right)
$$

Se essas informações estiverem disponíveis, será possivel trabalhar com uma função objetivo mais abrangente, que considere as perdas globais, qual seja:

$$
C_{G}(i)=C_{Q}(i)+C_{M}(i)
$$

Dessa forma, a otimização irá contemplar não apenas os custos devido a má qualidade de um produto, mas sim os custos globais, que incluem também os custos de matéria-prima e energia gastos na fabricação do produto. Caten e Ribeiro (1995) apresentam um estudo de caso que aborda esse aspecto.

\section{Etapa 5 - Otimização}

Essa etapa contempla a otimização propriamente dita, que consiste em identificar o ajuste que melhor atende simultaneamente o conjunto de variáveis de resposta. O ajuste ótimo corresponde ao ajuste que minimiza a função de perda quadrática multivariada. Ou seja, o ajuste ótimo é aquele que incorre na menor perda global.

Conhecidos os modelos para as variáveis de resposta, podemos escrever $Z=f(X)$, uma ve que $Z=f(Y)$ e $Y=$ $f(X)$. Usando o modelo $Z=f(X)$, o ajuste ótimo global para os fatores controláveis, pode ser identificado utilizando-sc, por exemplo, rotinas de programação linear ou não linear. Outra possibilidade ć simplesmente calcular $Z$ para diferentes tratamentos, isto é, diferentes ajustes dos fatores controláveis, e identificar o ajuste que minimiza $Z$.

Muitas vezes, a solução analítica para a função de perda, $Z=f(X)$, pode incorporar muitos termos. Nesses casos, uma alternativa interessante é utilizar, por 


\section{PRODUÇĀO}

exemplo, rotinas de regressão múltipla para obter um meta-modelo (ou seja, um modelo do modelo), relativamente simples, que se ajuste aos valores de perda estimados pela função completa para diferentes tratamentos. O meta-modelo, em geral, irá incorporar um número relativamente reduzido de termos, e isso facilita a interpretação e a análise gráfica dos resultados.

A análise gráfica pode ser realizada de diversas maneiras, como por exemplo, através de gráficos onde são plotadas curvas de isovalores para a função de perda em função de dois dos fatores controláveis. Nesse tipo de gráfico, podese visualizar como se comporta a função de perda dentro do intervalo dos fatores controláveis ensaiados no experimento. Com isso, pode-se visualizar regiões de mínima e máxima perda e a natureza de possiveis interações entre os fatores controláveis.

A dificuldade desse tipo de análise surge quando o número de fatores controláveis é grande. Nese caso, será necessário plotar e analisar vários gráficos.

Outra possibilidade de análise gráfica é a realização de um estudo de sensibilidade aos fatores controláveis. Para isso, altera-se um fator controlável dentro do intervalo ensaiado no experimento, enquanto mantêm-se os demais fatores fixos no ajuste ótimo, e observam-se as alterações no valor da função de perda. Esse tipo de análise gráfica é recomendável quando o estudo contempla muitos fatores controláveis. No entanto, a análise de sensibilidade não permite identificar interações entre os fatores controláveis, pois o efeito de um fator controlável sobre a função de perda é calculado enquanto os demais fatores estão fixos em seus ajustes ótimos.

A análise gráfica dos resultados permite identificar um envelope operacional para os fatores controláveis. O envelope operacional corresponde a um intervalo dos fatores controláveis que assegura valores relativamente baixos para a função de perda. Logo, o ajuste dos parâmetros pode variar dentro desse intervalo que a perda global decorrente será pequena.

\section{3- Estudo de Caso e Discussão}

Nesta seção é apresentado um estudo de caso que trata da otimização de um processo de fundição. Este estudo foi primeiramente apresentado por Chang e Shivpuri (1995). Será feita uma comparação entre os resultados obtidos por estes autores e aqueles obtidos utilizando-se o método proposto neste artigo.

\section{Etapa 1: Identificação do Problema}

Chang e Shivpuri (1995) fizeram uma boa discussão sobre as caracteristicas de qualidade de interesse no estudo de otimização. Segundo eles, atualmente a indústria tem enfrentado uma demanda dos consumidores por alta qualidade de fundição associada com baixos preços. No entanto, muitas vezes aumentar a 


\section{PRODUÇÃO}

qualidade de fundição pode resultar no desgaste da matriz de fundição. Esse é o caso quando a qualidade de fundição é conseguida por altas temperaturas de fusão, alta velocidade de entrada ou pequeno ciclo de tempo.

Uma vez que produzir ou reparar uma matriz é muito caro, o ganho pelo aumento da qualidade de fundição pode não compensar a perda pela redução do tempo de uso da matriz.

O que se pretende nesse estudo é encontrar o ajuste ótimo dos parâmetros do processo de fundição considerando simultaneamente a qualidade das peças fundidas e o tempo de uso da matriz. Desta forma, temos um processo com múltiplas variáveis de resposta de interesse, e a solução ótima implica em um compromisso entre elas.

Segundo Chang e Shivpuri (1995) existem duas categorias para as variáveis de interesse no estudo de otimização. A primeira refere-se à qualidade da fundição e a segunda ao tempo de uso da matriz. Exemplos de aspectos referentes à qualidade da fundição são as caracteristicas dimensionais e a porosidade do material fundido.

A variável de resposta escolhida para representar a caracteristica dimensional do material fundido foi a diferença entre o valor medido e o valor ideal do diâmetro do furo. Esta variável será chamada de Diferença de Diâmetro. É uma variável de resposta do tipo menor-é-melhor, e a unidade utilizada é o milimetro (mum).

A caracteristica de qualidade Porosidade relaciona-se com os pequenos vazios no material fundido, que surgem devido ao ar preso durante o processo de enchimento da matriz. A variável de resposta escollida para representar a porosidade foi a quantidade de ar liberada em um ensaio destrutivo onde a peça é derretida. Esta variável será designada por Porosidade. Trata-se de uma variável de resposta do tipo menor-é-melhor, e a unidade utilizada é centímetros cúbicos (cc) de vazios por 1000 gramas de material fundido.

A variável de resposta associada com o tempo de uso da matriz será a Diferença de Temperatura na superficie da cavidade da matriz. Sabe-se que quanto maior a diferença de temperatura maior a chance de formar fendas na superfície devido a fadiga térmica. É uma variável de resposta do tipo menor-é-melhor e a unidade da diferença de temperatura é

\begin{tabular}{|l|c|c|c|c|}
\hline Variáveis de resposta & Unidade & Tipo de C.Q. & Imp. relativa & Valor alvo \\
\hline $\mathrm{Y} 1=$ Diferença de diâmetro & $\mathrm{mm}$ & Menor-é-melhor & 1 & 0 \\
\hline $\mathrm{Y} 2=$ Porosidade & ce & Menor-é-melhor & 1 & 0 \\
\hline $\mathrm{Y3}=$ Diferença de temperatura & oF & Menor-é-melhor & 1 & 0 \\
\hline
\end{tabular}

Tabela 1 - Descrição das Variáveis de Resposta Utilizadas no Estudo Processo de Fundição 
Farenheit $\left({ }^{\circ} \mathrm{F}\right)$. A Tabela 1 apresenta a descrição das variáveis de resposta.

\section{Etapa 2: Plancjamento e Projeto de Experimentos}

Chang e Shivpuri (1995) primeiramente identificaram quais os parâmetros do processo que são mais influentes sobre as três variáveis de resposta escolhidas. Dentre os parâmetros do processo foram selecionados dois para serem investigados como fatores controláveis no experimento: temperatura do forno e tempo de permanênciada matriz.

A amplitude do intervalo dos fatores controláveis foi escolhida após discussão com os engenheiros responsáveis pelo processo de fundição. Os engenheiros concluíram que um modelo de primeira ordem não se ajustaria bem às variáveis de resposta Y1 e Y2. Em função dessa constatação, escolheu-se estudar cada um dos fatores controláveis a três niveis, possibilitando dessa forma o ajuste de modelos de primeira e segunda ordem aos dados coletados. A Tabela 2 apresenta os fatores controláveis e seus respectivos níveis ensaiados.

O modelo de experimento escolhido foi um projeto fatorial $3^{2}$ que permite avaliar dois fatores controláveis a três níveis cada um deles. A Tabela 3 apresenta os tratamentos ensaiados e os valores medidos para as variáveis de resposta.

\section{Etapa 3: Modelagem Individual das Variáveis de Resposta}

Essa é uma etapa muito importante, pois a modelagem individual das variáveis de resposta permite fazer estimativas dos valores de cada resposta para qualquer ajuste dos fatores controláveis.

Chang e Shivpuri (1995) realizaram a modelagem individual das variáveis de resposta. Com a intenção de aumentar os coeficientes de determinação da regressão, eles incluíram nos modelos das variáveis de resposta termos não significativos. Esse procedimento não se justifica uma vez que o coeficiente de determinação já era suficientemente alto e a inclusão de

\begin{tabular}{|l|c|c|c}
\hline \multicolumn{1}{|c|}{ Fatores Controliveis } & Nivel -1 & Nivel 0 & Nivel +1 \\
\hline $\begin{array}{l}\mathrm{X} 1=\text { Temperalura do } \\
\text { fonxo }\end{array}$ & $1250 \mathrm{oF}$ & $1300 \mathrm{oF}$ & $1350 \mathrm{oF}$ \\
\hline $\begin{array}{l}\mathrm{X} 2=\text { Tampo de } \\
\text { pernanêncin }\end{array}$ & $6 \mathrm{~s}$ & $7 \mathrm{~s}$ & $8 \mathrm{~s}$ \\
\hline
\end{tabular}

Tabela 2 - Fatores Controláveis Escolhidos para o Estudo do Processo de Fundição 
PRODUÇÃO

\begin{tabular}{|c|c|c|c|c|c|}
\hline Ensaio & $\begin{array}{c}\text { X1: } \\
\text { Temperatura } \\
\text { do forno }\end{array}$ & $\begin{array}{c}\text { X2: } \\
\text { Tempo de } \\
\text { permanência }\end{array}$ & $\begin{array}{c}\text { Y1: } \\
\text { Diferença de } \\
\text { diâmetro }\end{array}$ & $\begin{array}{c}\text { Y2: } \\
\text { Porosidade }\end{array}$ & $\begin{array}{c}\text { Y3: } \\
\text { Dif. de } \\
\text { temperatura }\end{array}$ \\
\hline 1 & -1 & -1 & 3 & 21 & 80 \\
\hline 2 & 0 & 0 & 7 & 17 & 95 \\
\hline 3 & 1 & -1 & 1 & 15 & 101 \\
\hline 4 & -1 & 0 & 8 & 19 & 85 \\
\hline 5 & 0 & -1 & 2 & 19 & 92 \\
\hline 6 & -1 & 1 & 4 & 20 & 87 \\
\hline 7 & 0 & 1 & 3 & 17 & 96 \\
\hline 8 & 1 & 0 & 5 & 14 & 106 \\
\hline 9 & 1 & 1 & 2 & 15 & 108 \\
\hline
\end{tabular}

Tabela 3 - Matriz Experimental e Resultados do Experimento $3^{2}$

termos não significativos não tem suporte estatístico.

Os modelos apresentados a seguir não são os mesmos apresentados por Chang e Shivpuri (1995) pois os termos não significativos não foram incluídos. As diferenças, no entanto, são pequenas, uma vez que a inclusão dos termos não significativos tem efeito pequeno sobre a estimativa da resposta.

Y1: Diferenç̧a do diâmetro do furo $\mathrm{YI}=6,67-1,167 \times \mathrm{X} 1+0,5 \times \mathrm{X} 2-4,167 \times \mathrm{X}^{2}$ $\mathrm{R}^{2}=0,98$

Y2: Porosidade $Y 2=16,67-2,67 \times \times 1-0,5 \times \times 2+1,167 \times \times 1^{2}$ $\mathrm{R}^{2}=0.96$
Y3: Diferença de temperatura

$Y=95,33+10,5 \times \times 1+3,0 \times \times 2-1,33 \times \times 1^{2}$

$\mathrm{R}^{2}=0,99$

Uma vez obtidos os modelos para cada uma das variáveis de resposta, pode-se encontrar os ajustes dos fatores controláveis que otimizam cada uma das variáveis de resposta separadamente. A Tabela 4 apresenta esses ajustes ótimos e os respectivos valores estimados para as variáveis de resposta.

Uma das vantagens da modelagem, é o fato de se poder estimar o valor das variáveis de resposta para niveis intermediários dos fatores controláveis. 
Além da modelagem das médias, também é possivel modelar a variabilidade das variáveis de resposta em função dos fatores controláveis. Com isso, pode-se ajustar o processo em níveis dos fatores controláveis que minimizem a variabilidade (tomem o processo robusto ao efeito dos fatores de ruido). No entanto, esse experimento não contempla repetições. Dessa forma, não se pode modelar a variabilidade a partir do desvio-padrão de cada tratamento. $E$, devido ao número reduzido de ensaios, fica dificil fazer uma análise de resíduos que eventualmente poderia indicar algum efeito dos fatores controláveis sobre a variabilidade.

\section{Etapa 4: Definição de uma Função Objetivo}

Chang e Shivpuri (1995) transfonnaram os modelos estimados $(Y i(x))$ em uma função utilidade $(\operatorname{di}(x))$, gerando valores entre $0 \mathrm{e} 1$. Para cada tipo de variável de resposta (menor-é-melhor, maior-é-melhor e nominal-é-melhor) existe uma transformação adequada de $Y i$ para di. A função utilidade é calculada de forma que, independente do tipo de variável de resposta, o valor alvo para $d i(0 \leq d i \leq 1)$ é sempre $d i$ $=1$. Ou seja, quanto mais próximo $d i$ estiver de 1, mais próximo a variável de resposta estará do seu valor ideal.

Uma vez calculada a função utilidade, a otimização simultânea das três variáveis de resposta é tratada como um problema dễ MODM (Multiple-Objective DecisionMaking Approach). A abordagem MODM propõe que o ajuste ótimo global seja encontrado da seguinte maneira:

Max di $(x), \quad i=1,2 \ldots \ldots . . \mathrm{r} \quad$ s.t. $\mathrm{x} \in$

intervalo operacional

que é equivalente a um problema de programacão não-linear:

Max $\alpha$ s.t. $\operatorname{di}(\mathrm{x}) \geq \alpha, \mathrm{i}=1,2, \ldots . \mathrm{r}, \mathrm{x} \in$ intervalo operacional

A magnitude de $\alpha$ pode indicar o desempenho do processo em relação às múltiplas respostas. Por exemplo, $\alpha=0,3$ indica que pelo menos uma das

\begin{tabular}{|c|c|c|c|c|c|}
\hline $\mathbf{Y}$ & $\mathbf{X 1}$ & $\mathbf{X 2}$ & $\mathbf{Y 1}$ & $\mathbf{Y 2}$ & $\mathbf{Y 3}$ \\
\hline $\mathrm{Y} 1$ & 1,000 & $-1,000$ & 0,845 & 15,66 & 101,51 \\
\hline $\mathrm{Y} 2$ & 1,000 & 0,214 & 5,42 & 13,95 & 106,41 \\
\hline $\mathrm{Y3}$ & $-0,972$ & $-0,974$ & 3,37 & 20,85 & 80,96 \\
\hline
\end{tabular}

Tabela 4 - Ajuste Ótimo dos Fatores Controláveis para cada Variável de Resposta Separadamente 


\section{PRODUÇÃO}

variáveis de resposta atinge apenas $30 \%$ do valor ótimo pretendido.

Em seu artigo, Chang e Shivpuri (1995) comparam os resultados obtidos utilizando-se a abordagem de MODM e os resultados obtidos utilizando-se uma outra abordagem proposta por Derringer e Suich (1980). Derringer e Suich (1980) propõem a maximização da média geométrica da função utilidade $\left(\mathrm{D}=(\mathrm{d} l \mathrm{~d} 2 \ldots \mathrm{dr})^{1 / r}\right)$ para encontrar o ajuste ótimo global.

Segundo Chang e Shivpuri (1995), a razão para o uso da abordagem MODM é que a solução conciliatória encontrada é mais equilibrada, ou seja, gera valores de di $(x)$ mais próximos um dos outros.

A função utilidade usada por Chang e Shivpuri (I995) já conduz parcialmente ao ajuste ótimo, pois penaliza os desvios do alvo das variáveis de resposta. A função utilidade não busca otimizar uma característica en detrimento das demais. $\mathrm{O}$ ajuste ótimo identificado pela função utilidade faz um compromisso entre as múltiplas variáveis de resposta, de forma que cada uma delas esteja relativamente próxima do valor ideal. No entanto, a função utilidade não tem uma ligação mais direta com custos da má qualidade; e tampouco incorpora outros aspectos importantes na otimização como a variabilidade das variáveis de resposta em torno do valor ideal, as possiveis oscilações dos fatores controláveis na produção e os custos de fabricação do produto.
Acredita-se que o critério mais adequado para se identificar o ajuste ótimo global seja a Função de Perda Quadrática Multivariada. A justificativa para seu uso será apresentada a seguir.

A performance de um produto/ processo deteriora-se gradualmente quando as variáveis de resposta desviamse do seu valor ideal ou quando há variabilidade em torno dele. Há um consenso na literatura de que a perda incorrida ao consumidor devido a má qualidade é, em muitos casos, aproximadamente proporcional ao quadrado do desvio do alvo. Esse modelo de comportamento é capturado pela Função de Perda Quadrática', cuja versão univariada foi proposta por Täguchi.

A função objetivo proposta neste artigo é uma versão multivariada da Função de Perda Quadrática. Ela possui as vantagens da Função de Perda (forte associação com aspectos econômicos perda financeira devido à má qualidade) e, além disso, pondera a importância relativa das várias variáveis de resposta $\mathrm{e}$ incorpora a preocupação com a variabilidade das variáveis de resposta e as possíveis oscilações nos fatores controláveis, em geral presentes durante a produão.

A Função de Perda Quadrática Multivariada aparece descrita em detallıe na primeira seção deste artigo (ver eq. 2). É importante ressaltar que a função objetivo proposta pode ser estendida para incluir outros aspectos econôninicos (além do aspecto referente à qualidade) no 
estudo de otimização (ver seção 2 , eq. 5). Dessa forma, custos de matéria prima ou custos de energia podem ser incluídos no estudo.

Em função dessas constatações, acredita-se que 0 emprego da Função de Perda Quadrática, usada há muito tempo pelos estatísticos (Desvio Quadrado Médio) e mais recentemente rediscutida por Taguchi e outros autores, seja o critério mais eficiente para dar suporte à busca do ajuste ótimo global.

Uma vez definida a função objetivo, os modelos individuais das variáveis de resposta são incluidos nessa função. Os coeficientes $w_{i}$ foram calculados atribuindo-se peso 1 para todas as variáveis de resposta e dividindo-se esse peso pelo quadrado da semi-amplitude das especificações. Uma vez que Chang e Shivpuri (1995) não comentam a respeito da importância relativa das diferentes variáveis de resposta, o objetivo dos coeficiente $w_{i}$, nesse caso, limila-se a climinar diferenças de escala entre as três variáveis de resposta.

Além disso, o modelo de experimento escolhido pelos autores não permite a modelagem da variabilidade das variáveis de resposta. Assim, não serì possível explorar ajustes robustos aos fatores de ruído. Chang e Shivpuri (1995) também não fornecem informações referentes às oscilações dos parâmetros do processo,de modo que não será possível explorar robustez às oscilações nos fatores controláveis.
Vale ressaltar que esses objetivos não serão incorporados devido à falta de informações disponiveis, uma vez que Chang e Shivpuri (1995) não se preocuparam com esses aspectos na otimização.

Em seu artigo, Chang e Shivpuri (1995) dizem que, após realizado o estudo de otimização, é necessário que o engenheiro tome uma decisão final de qual o melhor ajuste, considerando a estabilidade do chão-de-fábrica e os aspectos econômicos. Ou seja, eles mesmos apontam as falhas da função objetivo empregada (a Função Utilidade).

Ao contrário da Função Utilidade, a Função de Perda Multivariada está vinculada a aspectos econômicos e incorpora os termos necessários para considerar a estabilidade do chão de fábrica. Assim, chega-se a um resultado final muito mais consistente e abrangente.

\section{Etapa 5: Otimização}

A partir da função de perda definida anteriormente, utilizaram-se rotinas de programação linear para identificar o ajuste ótimo global. O ajuste ótimo global é aquele que minimiza a função de perda. A Tabela 5 apresenta uma comparação ęntre os ajustes ótimos encontrados por Chang e Shivpuri (1995), Derringer e Suich (1980) e pelo método proposto neste artigo.

Como pode-se verificar, o ajuste ótimo encontrado pelo método proposto neste artigo incorre no menor valor de perda. 


\section{PRODUÇÃO}

A perda gerada pelo ajuste ótimo encontrado pelo método proposto é aproximadamente 1,3 vezes menor do que o método de Chang e Shivpuri (1995) e 2,6 vezes menor do que a perda gerada pelo método de Derringer e Suich (1980).

A função utilidade usada no artigo de Chang e Shivpuri (1995) otimiza o processo considerando apenas a perda referente ao desvio das variáveis de resposta do seu valor alvo. Essa função utilidade já é um passo em direção do ajuste ótimo, uma vez que ela busca um compromisso equilibrado das múltiplas variáveis de resposta. No entanto, ela não considera a perda gerada pela variabilidade das variáveis de resposta (fruto do efeito dos fatores de ruído ) pelas oscilações dos fatores controláveis no chão-de-fábrica.

Ainda, a função utilidade usada por Chang e Shivpuri (1995) não contempla a incorporação de aspectos econômicos na identificação do ajuste ótimo global. Dessa forma, o ajuste ótimo global identificado pela função utilidade necessita de uma avaliação posterior dos engenheiros a respeito dos aspectos que não estão incorporados na função utilidade.

\section{4- Conclusões}

O método proposto neste artigo é quantitativo, flexível e genérico.

Quantitativo, pois todas as informações a respeito do produto ou processo são modeladas quantitativamente e 0 ajuste ótimo é encontrado por critérios numéricos.

Flexível, uma vez que permite que se trabalhe com várias caracteristicas de qualidade de interesse do consumidor e

\begin{tabular}{|l|cc|c|c|c|c|}
\hline \multicolumn{1}{|c|}{ Método } & $\mathbf{X 1}$ & $\mathbf{X 2}$ & $\mathbf{Y 1}$ & $\mathbf{Y 2}$ & $\mathbf{Y 3}$ & $\mathbf{Z}$ \\
\hline $\begin{array}{l}\text { Método } \\
\text { proposto }\end{array}$ & 0,345 & $-1,00$ & 1,61 & 17,41 & 94,64 & 0,254 \\
$\begin{array}{l}\text { Change } \\
\text { Shivpuri }\end{array}$ & $\mathbf{0 , 1 9}$ & $-0,78$ & 3,53 & 17,43 & 94,10 & 0,331 \\
\hline $\begin{array}{l}\text { Derringer e } \\
\text { Suich }\end{array}$ & 0,84 & $-1,00$ & 0,58 & 15,96 & 100,26 & 0,652 \\
\hline
\end{tabular}

Tahela 5 - Comparação entre os Ajustes Ótimos Encontrados pelos Diferentes Métodos 
do produtor, com importâncias relativas diferenciadas. Outro fator que contribui para a flexibilidade do método é a possibilidade de incorporar na otimização aspectos referentes à qualidade de um produto ou processo, como desvios do alvo, robustez ao ruído e às osciliações dos parâmetros do processo, assim como aspectos referentes aos custos de fabricação.

Genérico, pois devido à flexibilidade do método, ele pode ser aplicado à otimização experimental de produtos e processos em qualquer tipo de indústia.

Outros autores da literatura propõem métodos de otimização experimental; no entanto apenas consideram subjetivamente aspectos importantes na otimização, como por exemplo estabilidade do chão-de-fábrica e custos de fabricação do produto.

A proposta deste artigo tem a vantagem de ser mais abrangente, modelando quantitativamente uma maior diversidade de aspectos e reduzindo as subjetividades do estudo de otimização.

\section{Referências Bibliográficas}

BOX, G. E. P., HUNTER, W. G. \& HUNTER, J. S. Statistics for experimenters. John Wiley, New York, 1978.

CATEN, C.S. Otimização de produtus e processos mediclos por múltiplas caracteristicas de qualidade. Dissertação de mestrado, PPGEP/UfRGS, Porto Alegre, Brasil, 1995.
CATEN, C.S. \& RIBEIRO, J.L. Custos da qualidade e da manufatura: um estudo de caso na indústria química. Relatório Técnico 06/95, PPGEP/ UFRGS, Porto Alegre, Brasil, 1995.

CHANG, I. S. \& SHIVPURI, R. A Multiple-Objective Decision-Making Approach for assessing simultaneous improvement in die life and casting quality in a die casting process. Quality Engineering, vol. 7, no 2, pp. 371-383, 1995.

COLEMANN, D. E. \& MONTGOMERY, D. C. A sistematic approach to planning for a design industrial experiment. Technometrics, February, vol. 35, no 1, pp. 1-12, 1993.

DERRINGER, G. \& SUICH, R. Simultaneous Optimization Response Variable. Journal of Quality Technology, vol 12, pp. 214-219, 1980.

HAHN, G. J. Some things engineers should know about experimental design. Journal of Quality Technology, vol. 9, no 1, pp. 13-20, 1977.

MONTGOMERY, D.C. Design and analysis of experiments. John Wiley and Sons, New York, 3nd ed., 1991.

NANNI, L. F. \& RIBEIRO, J.L. Planejamento e avaliação de experimentos. Caderno de Engenharia 17/87, CPGEC/UFRGS, Porto Alegre, Brasil, 1991. 


\section{PRODUÇÃO}

RIBEIRO, J. L. \& ELSAYED, E. A. A case study on process optimization using the gradient loss function. International J. of Production Research, vol. 33, no. 12, pp. 3233-3248, 1995.

Artigo recebido em abril/1996, avaliado em junho/1996 e aprovado para publicaçåo em julho/1996 